\title{
Role of relativity and nucleon compositeness in few-body systems
}

\author{
S. J. Wallace ${ }^{a *}$

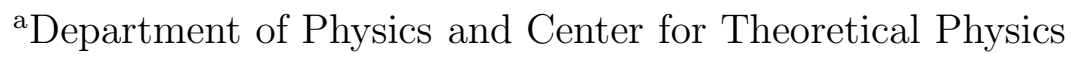 \\ University of Maryland, College Park MD 20742-4111
}

Recent progress is reviewed in four areas where new experimental data have been obtained: proton-proton bremsstrahlung, threshold pion production in proton-proton collisions, elastic electron-deuteron scattering and deuteron photodisintegration at several GeV photon energy. Relativistic effects are expected to be significant in these processes. High energy photodisintegration suggests that partonic behavior could be relevant in subamplitudes at large momentum transfer.

\section{INTRODUCTION}

This review of recent work in the field of few-body systems follows an earlier review [1] in focusing on particular experiments whose interpretations are expected to involve relativistic effects along with other physics. Relativistic effects generally are expected to be significant in the dynamics of few-body systems when one or more momenta involved are comparable to the nucleon's mass. Even in cases where a nonrelativistic interpretation is successful, a relativistic treatment is preferred provided that it contains all the relevant physics. These points motivate much work in the field, but it must be recognized that identifying a relativistic effect can be a subtle matter.

Depending upon the form and organization of the dynamics, relativistic effects may take different forms. For example, there are several ways to reduce the four-dimension BetheSalpeter equation to three dimensions. 20 5] Matrix elements corresponding to physical observables should be the same independent of which reduction is used provided that equivalent lagrangians are used and all relevant terms are kept. Effects in one form of dynamics, such as Z-graphs or off-shell effects in a subamplitude or short-range mesonexchange currents, may be replaced by contact terms in another form of dynamics, with similar results. Effects of boosts may be in the wave functions in one analysis and in the currents in another, again with similar results for matrix elements. It is essential to understand these differences in order to see how pieces of the physics get shuffled from one place to another by the choice of formalism. Equivalent results should be found if each formalism is fully evaluated. Relativistic effects can be different depending on the formalism used.

Although different forms of dynamics applied to the same lagrangian should produce equivalent results, this fails if currents are not consistent with the interactions between the particles. One of the main points of relativistic formulations based on a meson-exchange

\footnotetext{
${ }^{*}$ Support of the U.S. Dept. of Energy under grant DE-FG02-93ER-40762 is gratefully acknowledged.
} 
lagrangian is to be able to apply Mandelstam's construction of currents [6] for the BetheSalpeter equation[7] in order to formulate currents that are consistent with interactions. It becomes interesting to discuss the physics differences between various analyses once this has been done, the goal being to discover a lagrangian that works to describe the physics independently of the formalism used to evaluate it.

In recent years, considerable attention has been given to the problem of formulating consistent currents. Arenhövel and collaborators have developed consistent currents for the analyses of electromagnetic interactions of the two-nucleon system by considering a rather complete set of $1 / M$ corrections to the nonrelativistic formalism. [8] Reductions of the Bethe-Salpeter equation to three-dimensions also require a reduction of the currents to three dimensions. Gross and Riska[9], and more recently Adam, Van Orden and Gross 10], and Blankleider and Kvinkhidze 11] have discussed the appropriate current for the spectator formalism, in which one particle is on its mass shell. The spectator formalism requires a careful consideration of the case where the photon is absorbed by the on-shell nucleon. Phillips and Wallace 12 have developed the appropriate currents for the equaltime formalism. An important result in Refs. [10,12] is that truncating the interaction at a definite order in the coupling constants, and truncating the exchange currents at the same order, preserves the Ward-Takahashi identities. This ensures that the formalism has conserved currents and is well suited to phenomenology based on the one-boson-exchange truncation. Works by Carbonell, Desplanques, Karmanov and Mathiot [13 and de Melo, Frederico, Naus and Sauer 14 have discussed the currents for light-front dynamics. These authors find that contact terms are required in the light-front formalism in order to replace Z-graphs of a covariant formalism. This is an example of a relativistic effect that takes a different form in different dynamics.

\section{BREMSSTRAHLUNG}

Recent experiments have provided precise bremsstrahlung data for proton-proton collisions using a $190 \mathrm{MeV}$ polarized proton beam.15,16 Data at $389 \mathrm{MeV}$ also have been obtained at RCNP Osaka [17] and an experiment at $300 \mathrm{MeV}$ [18 has been performed at COSY in order to check older TRIUMF measurements. 19 At the same time, questions have been raised about the motivations for studying the bremsstrahlung process. 20 22]

Historically, bremsstrahlung experiments have been motivated by a desire to access off-shell matrix elements of the $N N$ interactions, with the expectation that this would help to discriminate between potential models that yield equivalent descriptions of the $N N$ data. Fearing 20,21 has pointed out that this motivation is flawed because off-shell effects are ambiguous. When one starts from a lagrangian, field transformations may be used to shuffle off-shell effects in one analysis into contact terms in another analysis that is based on an equivalent lagrangian. 22 Equivalence means that all S-matrix elements are the same for the two lagrangians. Off-shell effects are not the same for equivalent lagrangians, which makes them ambiguous and unmeasurable.

The arguments of Fearing and Scherer do not take into account a standard convention that constrains the ambiguity in nucleon-nucleon bremsstrahlung. Generally, models of the $N N$ interaction are based on an integral equation with a two-nucleon Green's function, $G$, and the Green's function is constrained to have a standard form when both 
nucleons are in positive-energy states. In order to maintain the standard form for $G$, interactions have to absorb the effects of field transformations. Otherwise, one would add nonstandard terms to $G$. Likewise, the currents for nucleons in positive-energy states are constrained to be consistent with the standard from of $G$, which requires the effects of field transformations to be absorbed into exchange currents and pair currents. Nevertheless, significant ambiguities remain.

One well-known source of ambiguity is the fact that there are unitary equivalences between interactions that reproduce the same $N N$ data. A second source of ambiguity arises because currents cannot be formulated consistently for phenomenological potential models because one does not have an underlying lagrangian. It is possible to prescribe a connection between the various spin- and isospin-dependent terms in the phenomenological $N N$ potential and the usual set of meson exchanges. Applying the Mandelstam procedure to these parts of the potential as if they were caused by meson exchanges provides a prescription for construction of currrents. 223 However, there is no clear way to avoid ambiguities in such a prescription. Because of the ambiguities, the motivation for studying bremsstrahlung has to be to find a lagrangian that works, as discussed in the Introduction.

Low's soft-photon theorem [24] shows that bremsstrahlung has large contributions at low photon momenta that are determined by the on-shell $N N$ scattering amplitudes and model-independent factors involving $k^{-1}$ and $k^{0}$, where $k$ is the photon momentum. The recent KVI experiments use a $190 \mathrm{MeV}$ proton beam and detect the two final-state protons, allowing a reconstruction of the bremsstrahlung photon. A photon energy of about $60 \mathrm{MeV}$ is typical and this is expected to provide sensitivity to effects beyond those controlled by the low-energy theorem. Experimental results have been compared with theoretical calculations of Martinus et al. [25,26] that are based on a relativistic mesonexchange model, including important contributions from $\Delta$ currents. The theoretical results of Martinus et al. differ significantly from the data at forward angles, but agree better at larger angles and for the analyzing power. The forward angle discrepancy seems to be because the $N N$ model used 27] does not provide accurate phase shifts at the relevant energies. Improvement of the description of phase shifts yields improved results for bremsstrahlung. [28] The experimental cross section data are in very good agreement with an analysis based on a soft-photon approximation. 29,30 However, the soft-photon approximation does not describe the analyzing power well. The soft-photon approximation takes into account terms beyond those controlled by Low's theorem because on-shell $N N$ amplitudes are used at energies and angles appropriate to both the initial and final states of the $N N$ system in the bremsstrahlung process.

The impressive precision of the new bremsstrahlung data from KVI makes it possible to detect interesting effects of relativistic dynamics, $\Delta$ currents and meson-exchange currents. Previous calculations of these effects 25, 31, 32 have shown them to be large compared with what can be resolved by the new data. It remains to be seen whether improved theoretical calculations will provide a good understanding of the new bremsstrahlung experiments. 


\section{THRESHOLD PRODUCTION OF NEUTRAL PIONS}

It is now ten years since precise experiments on threshold production of $\pi^{0}$ mesons were performed using a cooled proton beam and a hydrogen target at the Indiana University Cyclotron Facility. [33,34] The surprise was that cross sections for $p p \rightarrow p p \pi^{0}$ were five times larger than existing theoretical predictions. 35, 36] A confirmation of the Indiana experiment was obtained at CELSIUS. 37]

Over the past ten years, there has been substantial theoretical interest in the $\pi^{0}$ production process. Theoretical calculations all have shown that the best understood mechanisms for soft pion production are suppressed in the reaction. This was confirmed by several groups using chiral perturbation theory [38 41] and is also found in phenomenological models. 42 44 Soft-pion contributions are small in part because of cancellations between the amplitude for direct pion emission and the rescattering amplitude in which the pion is emitted by one nucleon and scatters from the other. An important point is that the proton momentum required for pion production is at least $\sqrt{m_{\pi} M} \approx 2.6 m_{\pi}$, which is not small in comparison with the pion mass. [38,41] A recent analysis 45] indicates that chiral perturbation theory converges poorly for the reaction. Because the soft pion contributions are much too small to explain the data, a new term must be added to the chiral lagrangian to sum up all effects of short-range pion production. This term is fit to the experimental data. 46.

As first pointed out by Lee and Riska [47], suppression of effects from soft pions allows smaller effects from short-range physics to become prominent. Lee and Riska showed that the data could be explained by including a short-range contribution to the axial charge operator, namely, pion production from an intermediate $N \bar{N}$ pair state that is produced by exchange of $\sigma$ and $\omega$ mesons. The importance of this short-range exchange current was confirmed in calculations by Horowitz and collaborators 48. However, both analyses used a perturbative treatment of the relativistic effects from Z-graphs. The validity of the perturbative approach to including short-range effects such as Z-graphs was questioned by Adam et al. [49] using the spectator formalism of Gross.

Various refinements and alternative mechanisms for threshold pion production have been proposed, such as couplings to the $N \Delta$ channels as intermediate states. This is motivated by a recoil contribution in the $N N \rightarrow N \Delta$ transition that can be significant near threshold [50]. Using a model of the $\pi N$ scattering amplitude that fits the experimental data, and includes off-shell effects, a Julich collaboration [42] reinvestigated the pionic mechanisms and $\Delta$-recoil contributions with the conclusion that the $\Delta$-recoil contribution is not large enough to explain the cross section data.

The major progress in the past few years has been the measurement of proton-spin observables in $\pi^{0}$ production near threshold at Indiana. 515 53] The experiments were designed to allow a determination the separate contributions of s-waves and p-waves, assuming these to be the only contributing partial waves. The results provide a better understanding of the reaction and of the role of the $\Delta$ isobar in it. The theoretical model of the Julich group [43,44 has been extended to allow calculations of spin observables also, however, no other calculations of spin observables are available.

The Julich group includes the standard pion emission, pion rescattering and pion production mechanisms involving intermediate $\Delta$ states. These ingredients produce a theo- 
retical cross section that is too small by about a factor of two. Including a short-range contribution from Z-graphs allows the cross section data to be fit by adjusting the strength of the short-range contribution. Moreover, the spin observables are also reasonably well described. The calculations indicate that for pion momenta below $0.8 m_{\pi}$, the $\Delta$ contribution to cross sections is small in comparison with the needed short-range contribution.

Data for spin observables show trends as pion momentum increases that are similar to trends in the calculations of the Julich group stemming from the p-wave contributions of the $\Delta$. However, the trends start at somewhat lower pion momentum in the data than in the theoretical calculations. This would be consistent with p-wave contributions being important somewhat closer to threshold than the theoretical model suggests. There has been a report of $\mathrm{D}$-wave production of $\pi^{0}$ near threshold based upon very precise measurements of angular distributions. 54

The clear understanding that has emerged over the last ten years of work is that s-wave threshold $\pi^{0}$ production requires a short-range contribution that may take one or another equivalent form, i.e., a pion-production contact term, or meson-exchange currents involving Z-graphs or other meson-exchange currents. An example of other meson-exchange currents is shown in the work of Riska and collaborators [55, who show that the $\rho \pi \omega$ exchange current can contribute significantly, but intermediate $N^{*}$ resonances provide a small contribution. Thus, the precise nature of the short-range contribution is not settled but all analyses show that there must be one. The $\pi^{0}$ production at threshold has provided a unique window to short-range effects in the $N N$ interaction.

\section{ELASTIC ELECTRON-DEUTERON SCATTERING}

Major experimental progress has been made in electron-deuteron scattering since the review at the Groningen meeting. Experiments have been completed at the Thomas Jefferson National Accelerator Facility that measure the deuteron's $A$ form factor and the tensor alignment parameter $t_{20}$ for $Q>1 \mathrm{GeV} / \mathrm{c}$. 56 58 New data for $B\left(Q^{2}\right)$ are expected to be published soon.

The large momentum transfers involved in recent experiments motivate relativistic treatments of the deuteron and its electromagnetic interactions. As noted in Ref. [1], measurements of the longitudinal-transverse asymmetry, $A_{\phi}$, in (e,e'p) reactions [59,60] have provided clear evidence that current matrix elements of a nucleon in a deuteron should be treated within a relativistic formalism that incorporates at least positive-energy Dirac spinors, or equivalent relativistic effects. The low-energy theorem of Refs. 61,62 shows that for a composite nucleon, there are contributions to second-order interactions from contact terms, off-shell effects and the composite-particle Z-graph. For scalar and vector interaction at low energy, the sum of such terms produces the same effect as is obtained from a Z-graph for an elementary particle. This suggests use of the Dirac propagator for a nucleon as an efficient means to include the model-independent effects. However, it is important to respect chiral symmetry when negative-energy components are included by using pseudovector $\pi N$ coupling.

Nonrelativistic analyses that include leading-order relativistic corrections also provide a consistent analysis for smaller $Q$, i.e., $Q \leq M$. A recent analysis of elastic electron scattering from the deuteron by Arenhövel, Ritz and Wilbois [63] is based on a meson-exchange 
model that takes into account a rather complete set of relativistic and exchange-current corrections. Essentially the same model has been used in analyses of electrodisintegration and photodisintegration of the deuteron. 64.8] It provides a particularly consistent framework for the low $Q$ regime.

Over the past ten years, there have been three relativistic analyses of $e+D$ elastic scattering based on quantum field theory, assuming an effective $N N$ interaction based on exchange of mesons. Hummel and Tjon 65 used a Blankenbecler and Sugar reduction to three dimensions, Gross, Devine and Van Orden [66] used the spectator formalism with one particle on mass shell, and Phillips, Wallace and Devine 67,68 have used an equal-time (ET) reduction to three dimensions. Although somewhat different relativistic equations are used in each analysis, they have many common features: each corresponds to a reduction of the Bethe-Salpeter equation to three dimensions and each incorporates Dirac-spinor wave functions for the interacting nucleons, including negative-energy components. With such wave functions, pair currents are included automatically when the impulse approximation current is calculated. Mandelstam's construction of the current [6] has been used to derive consistent currents for the three-dimensional reductions and this makes them attractive theoretically.

Light-front calculations have been performed for electron-deuteron scattering recently by Carbonell and Kharmanov. 69 In this case it is necessary to include contact terms in order to reproduce the effects of pair currents. In principle, it should be possible to define the consistent currents for the light-front approach similarly to what has been done for the other formalisms that are based on quantum field theory. An alternative approach to light-front calculations is provided by Chung et al. [70] Whether or not exchange currents can be constructed in this approach so as to achieve equivalence with the others is an open question.

The most consistent relativistic calculations based on a meson-exchange lagrangian have been performed by Gross, Devine and Van Orden. 66 Coupling constants and other parameters of the meson-exchange model were fit so as to achieve a good description of the modern $N N$ phase shifts. 77]

There is rather good agreement between four relativistic impulse approximation calculations and the new $t_{20}$ data from Jefferson Laboratory. The relativistic calculations of Hummel and Tjon 65, Van Orden, Devine and Gross 66 and Phillips, Wallace and Devine 67] all show that the $t_{20}$ data are well described by the impulse approximation. Similarly good agreement has been obtained by Carbonnel and Karmanov using the lightfront formalism. 69

For $A(Q)$, the new data extend to $Q^{2}=6(\mathrm{GeV} / \mathrm{c})^{2}$. Relativistic calculations based on the impulse approximation underpredict the $A\left(Q^{2}\right)$ data at large $Q$. However, calculations of Van Orden, Devine and Gross that include the $\rho \pi \gamma$ meson-exchange current contribution are in good agreement with the data. The calculations include the $\rho \pi \gamma$ exchange current using a soft form factor, $F_{\rho \pi \gamma}\left(Q^{2}\right)$, for the $\rho \pi \gamma$ vertex that is motivated by a quark model. Calculations of Hummel and Tjon used a vector-meson dominance model of the $\rho \pi \gamma$ form factor that does not fall as rapidly with increasing $Q^{2}$, which results in $A\left(Q^{2}\right)$ being too large at high $Q^{2}$. Thus, the softer $\rho \pi \gamma$ form factor is favored by the $A\left(Q^{2}\right)$ data.

Three types of meson-exchange currents can play a role in the electron deuteron scat- 
tering, namely, $\rho \pi \gamma, \omega \sigma \gamma$, and $\omega \eta \gamma$ currents. In the calculations of Hummel and Tjon, Van Orden, Devine and Gross and Phillips, Wallace and Devine, inclusion of the $\rho \pi \gamma$ current gives a rather small effect in $t_{20}$ that tends to improve the agreement with data slightly. However, the $\omega \sigma \gamma$ meson-exchange current that has been considered by Hummel and Tjon is ruled out by the new $t_{20}$ data. It predicts a significant shift of the impulse approximation result towards higher $Q$ that is not consistent with experiment. Contributions from the $\omega \eta \gamma$ current are rather small and they may be omitted. Thus, the new $t_{20}$ data do not indicate much importance for meson-exchange contributions except at the highest $Q$. This is very interesting because the meson-exchange currents, particularly the $\omega \sigma \gamma$ current, contain substantial uncertainties.

Because the $\omega \sigma \gamma$ current is uncertain, it has been omitted in many analyses. It was introduced by Hummel and Tjon in order to achieve a reasonable description of the magnetic form factor, $B(Q)$, which is poorly described either by the relativistic impulse approximation or when the $\rho \pi \gamma$ exchange current is included. There is a surprising effect, first pointed out by Zuilhof and Tjon 72], that causes relativistic calculations of the magnetic form factor to deviate from the data. When the $\rho \pi \gamma$ current is included, the standard form of the coupling of the $\rho$-meson to the nucleon, i.e., $g_{\rho N N}\left(\gamma^{\mu}+\kappa / M \sigma^{\mu \nu} q_{\nu}\right)$, arises as a factor in the $\rho \pi \gamma$ exchange current between two nucleons. In a $p / M$ expansion, the tensor term proportional to $\kappa$ is higher order and often is omitted. 23,63. However, the tensor term can be important because $\kappa \approx 7$, making the tensor coupling of relative order $Q / m_{\pi}$, rather than $Q / M$. In relativistic calculations, the tensor term generally is included. The result is that there is a sign change of the $\rho \pi \gamma$ exchange-current contribution to the magnetic form factor near $Q^{2} \approx 1(\mathrm{GeV} / \mathrm{c})^{2}$. This sign change occurs at a lower $Q^{2}$ than the minimum of the magnetic form factor, which is near $Q^{2} \approx 2(\mathrm{GeV} / \mathrm{c})^{2}$. Because of the sign change, the $\rho \pi \gamma$ current shifts the minimum of $B\left(Q^{2}\right)$ to lower $Q$. Without the sign change, the $\rho \pi \gamma$ exchange current shifts the minimum of $B\left(Q^{2}\right)$ toward higher $Q$, which agrees better with the data. This effect has now been seen in a number of calculations. Hummel and Tjon incorporate the tensor term, with a consequent shift of $B(Q)$ away from the data, and Phillips, Wallace and Devine 68 have reproduced the effect using the same $\rho \pi \gamma$ current. Recent calculations by Van Orden also show the same effect when the $\rho \pi \gamma$ current is included. Schiavilla 73 has independently checked this and has seen the same effect.

The sign change in the $\rho \pi \gamma$ contribution would not have an adverse effect if it were to occur at higher $Q$, for example, $Q^{2}>2(\mathrm{GeV} / \mathrm{c})^{2}$. A smaller ratio of $\rho N N$ tensor to vector coupling would help to move the sign change to higher $Q^{2}$.

Because the new $t_{20}$ data seem to rule out a significant contribution from the $\omega \sigma \gamma$ current, some other contribution is required in order to explain the magnetic form factor. It has been found by Gross, Devine and Van Orden that Z-graph contributions are large enough to provide an explanation. Including the negative-energy components of the deuteron's wave function, as calculated in the spectator formalism, provides a substantial shift of the calculated minimum of the $B(Q)$ form factor towards higher $Q$. Attempts to confirm this result by Phillips and Wallace and also by Tjon have not been successful. The ET calculations show only rather small effects of negative-energy components and no significant shift of the minimum of $B\left(Q^{2}\right)$. This is surprising because the ET propagator provides a factor of two enhancement of negative-energy components in comparison with 
the symmetrized propagator of the spectator formalism, simply because the one-body limit of the ET propagator gives the Dirac propagator for each of the two nucleons. Thus, negative-energy components should be larger in ET calculations than in spectator calculations, other things being equal. Perturbative calculations have verified the small effects of Z-graphs in ET calculations. Thus, there is no convergence regarding the explanation of $B(Q)$ and further work on the subject is warranted.

The deuteron form factor as defined by $F_{d}\left(Q^{2}\right) \equiv \sqrt{A\left(Q^{2}\right)}$ is observed to decrease as $Q^{-10}$ at large $Q$. 577. This simple scaling behavior has been predicted from perturbative QCD, but generally $Q$ is thought to be too low for the perturbative QCD explanation to be valid. [74, [5] Similar behavior can be obtained from a meson-exchange calculation as indicated by the successful description of $A\left(Q^{2}\right)$ by Van Orden and Gross.

In summary the relativistic impulse approximation calculations provide good agreement with the $t_{20}$ data up to $Q^{2} \approx 1.5(\mathrm{GeV} / \mathrm{c})^{2}$. The data for $A\left(Q^{2}\right)$ up to $Q^{2} \approx 6(\mathrm{GeV} / \mathrm{c})^{2}$ can be described when the $\rho \pi \gamma$ exchange current is included. Results for $B\left(Q^{2}\right)$ form factor show much larger deviations from the data, indicating that a new contribution may be needed in the theoretical models. There is not yet a convergence on what the new contribution is.

\section{PHOTODISINTEGRATION OF THE DEUTERON}

Very interesting experiments at Jefferson Laboratory have measured cross sections and induced polarization in photodisintegration of the deuteron with photons up to $4 \mathrm{GeV}$. [76] The initial results were for final state nucleons at $\theta_{c . m .}=36^{\circ}, 52^{\circ}, 69^{\circ}$ and $89^{\circ}$, where $\theta_{c . m}$. is the angle between the final-state proton and neutron in their center of mass. At the largest angle, cross sections exhibited scaling behavior as a function of the invariant mass squared $s=4 M^{2}+2 M E_{\gamma}$, i.e., $s^{11} d \sigma / d t \approx$ constant. As the angle between the nucleons was decreased, the cross sections deviated from scaling behavior. One way to understand this is in terms of the momentum transfer $r$ in the diagram shown in Fig. 1 which depicts a generic exchange mechanism between the two nucleons. Approximating the two nucleons in the initial-state deuteron as each having zero momentum leads to a simple expression for the momentum transfer, i.e., $r^{2}=-M E_{\gamma}\left(1-\alpha \cos \theta_{c . m}\right)$, where $\alpha=1 / \sqrt{1+M / E_{\gamma}}$. Thus, the momentum transfer is spacelike and is largest in magnitude when $\theta_{c . m .}=90^{\circ}$. It decreases rapidly as the angle becomes smaller as shown in Fig. 2. At $\theta_{\text {c.m. }}=36^{\circ}$, the smallest angle in the experiment, momentum transfer is not greater than about 1 $(\mathrm{GeV} / \mathrm{c})^{2}$. For $E_{\gamma}=4 G e V$ and $\theta_{c . m} .=90^{\circ}, r^{2} \approx-3.7(G e V / c)^{2}$. Thus the experiment spans a large range of momentum transfer between the proton and neutron.

The most recent results show that the induced polarization of the proton tends to zero when the photon energy is greater than $1 G e V$ at $\theta_{c . m .}=90^{\circ}$. [77 Polarization transfer observables tend to zero for $E_{\gamma}>2 G e V$. Thus, at the largest momentum transfers in the experiment, there is evidence that the process is consistent with helicity conservation. A theoretical explanation of the cross section data has been developed by Frankfurt, Miller, Sargsian and Strikman 78 by relating the process to a hard pn scattering process.

The photodisintegration reaction probes interactions over a large range of momentum transfers. At lower momentum transfer, it is expected to be dominated by hadronic interactions. At the highest momentum transfer, partonic interactions may be important 


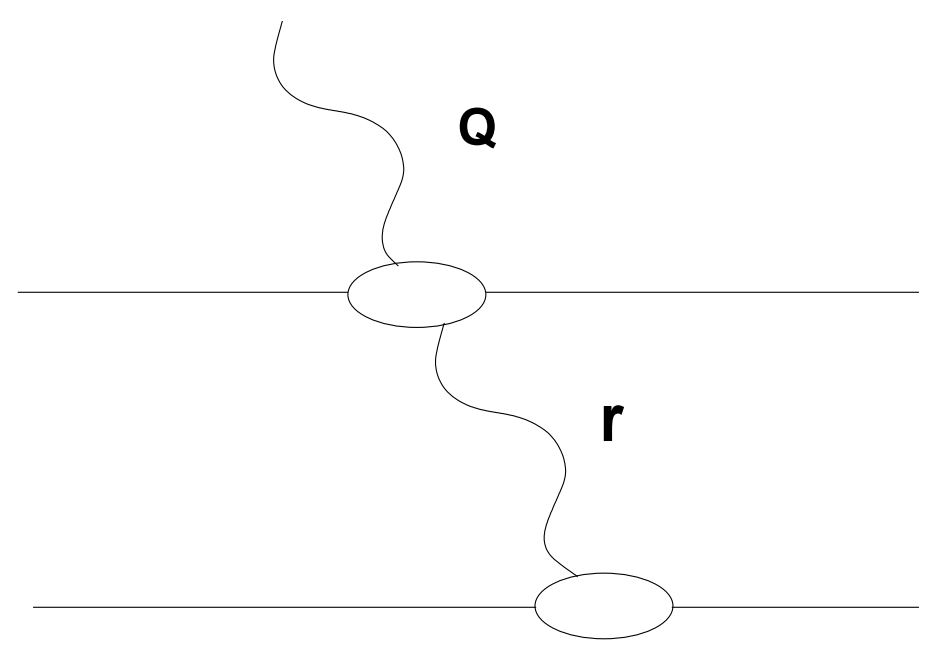

Figure 1. Photon absorption followed by meson exchange between the nucleons in a deuteron

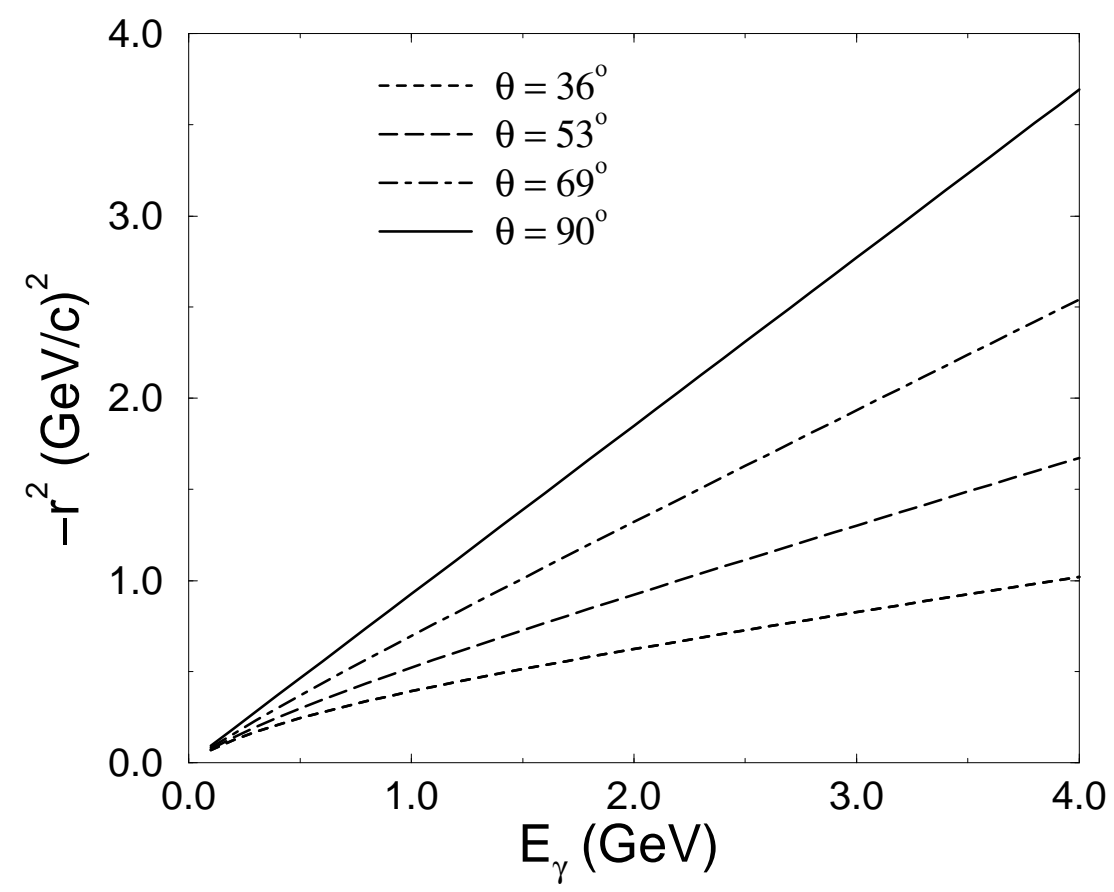

Figure 2. Variation of momentum transfer with photon energy. 
in the subamplitude where the photon is absorbed. A very interesting question is whether a transition occurs from hadronic to partonic interactions within the range of photon energies considered in the recent experiments.

\section{SUMMARY}

Very precise data have been obtained for the bremsstrahlung process in $p p$ collisions. They are sufficient to explore interesting effects of relativity and meson-exchange currents and to determine whether the conventional meson-exchange lagrangian is adequate. Theoretical calculations based on dynamical models or on soft-photon approximations do not yet provide a consistent understanding of the new data.

Progress in threshold production of $\pi^{0}$ mesons in $p p$ collisions has been made by measuring spin observables, which allow a separation of s-wave and p-wave parts of the amplitude. At energies close to threshold where the s-wave contributions dominate, experimental cross sections are about a factor of two larger than the most complete theoretical cross sections, which are based on contributions of soft pions and intermediate $\Delta$-states. A short-range contribution is need to provide the missing strength. The exact nature of the short range contributions is still debated, but it is consistent with being a mixture of relativistic effects and short-range meson-exchange currents.

New data from Jefferson Laboratory have provided indications that relativistic models based on meson-exchange forces work surprisingly well to large $Q^{2}$. Particularly the $t_{20}$ data show that short-range meson-exchange currents must play a fairly small role up to $Q^{2} \approx 1.5(\mathrm{Gev} / \mathrm{c})^{2}$. The $\rho \pi \gamma$ exchange current is needed at large $Q^{2}>2-3(\mathrm{Gev} / \mathrm{c})^{2}$ to describe the $A\left(Q^{2}\right)$ data, but the calculations based on the relativistic impulse approximation are reasonable for $A\left(Q^{2}\right)$ and quite close to the experimental data for $t_{20}$. However, there is a puzzle regarding the magnetic form factor. The contribution of the $\rho \pi \gamma$ exchange current that helps for $A\left(Q^{2}\right)$ worsens the agreement between theory and experiment for $B\left(Q^{2}\right)$. Relativistic effects could make a significant contribution, but the existing analyses provide a conflicting assessment of their role. Thus, a consistent picture has not yet been established.

Intriguing experimental results have been obtained for deuteron photodisintegration at photon energies up to $4 \mathrm{GeV}$. Cross sections exhibit scaling behavior at the largest momenta of the experiment, but these momenta are too low for perturbative QCD to be valid. Possibly a subamplitude of the process becomes dominated by partonic interactions at the momentum transfers involved. For example, the initial photon absorption by a nucleon might be dominated by a partonic amplitude, followed by a meson exchange to share the momentum transfer with the second nucleon. A variety of theoretical possibilities should be explored in order to understand the deuteron photodisintegration reaction and to investigate a possible transition from hadronic interactions to partonic interactions at high photon energy.

\section{REFERENCES}

1. S. J. Wallace, Nucl. Phys. A631 (1998) 137c.

2. A. A. Logunov and A. N. Tavkhelidze, Nuovo Cimento 29 (1963).

3. R. Blankenbecler and R. Sugar, Phys, Rev. 142 (1966) 1051. 
4. F. Gross, Phys. Rev. 184 (1969) 1448; Phys. Rev. C26 (1982) 2203.

5. D. R. Phillips and S. J. Wallace, Phys. Rev. C54 (1996) 507.

6. S. Mandelstam, Proc. Roy. Soc. (London) A233 (1955) 248.

7. E. E. Salpeter and H. A. Bethe, Phys. Rev. 84 (1951) 1232.

8. F. Ritz, H. Göller, T. Wilbois, and H. Arenhövel, Phys. Rev. C55 (1997) 2214.

9. F. Gross and D. O. Riska, Phys. Rev. C36 (1987) 1928.

10. J. Adam, Jr., J. W. Van Orden and F. Gross, nucl-th/9710009.

11. A. N. Kvinikhidze and B. Blankleider, Nucl. Phys. A 631, (1998) 559c.

12. D. R. Phillips and S. J. Wallace, Few Body Sys. 24 (1998) 175.

13. J. Carbonell, B. Desplanques, V. A. Karmanov and J.-F. Mathiot, Phys. Rep. 300 (1998) 215.

14. J. P. B. C. de Melo, T. Frederico, H. W. L. Naus and P. U. Sauer, nucl. Phys. A660 (1999) 219.

15. H. Huisman et al., Phys. Rev. Lett. 83 (1999) 4017.

16. H. Huisman, et al., Phys. Lett. B476 (2000) 9.

17. K. Yasuda et al. Phys. Rev. Lett. 82 (1999) 4775.

18. R. Bilger et al., Phys. Lett. B429 (1998) 195.

19. K. Michaelian et al., Phys. Rev. D41 (1990) 2689.

20. H. W. Fearing, Phys. Rev. Lett. 81 (1998) 758.

21. H. Fearing, Few Body Systems Suppl. 0 (2000) 1.

22. H. W. Fearing and S. Scherer, Phys. Rev. C62 (2000) 034003.

23. D. O. Riska, Phys. Rep. 181 (1989) 207.

24. F. E. Low, Phys. Rev. 110 (1958) 974.

25. G. H. Martinus, O. Scholten and J. A. Tjon, Phys. Rev. C56 (1997) 2945.

26. G. H. Martinus, O. Scholten and J. A. Tjon, Phys. Rev. C58 (1998) 686; Phys. Lett. B402 (1997) 7.

27. J. Fleischer and J. A. Tjon, Nucl. Phys. B84 (1974) 375; Phys. Rev. D15 (1977) 2537; Phys. Rev. D21 (1980) 87.

28. J. A. Tjon, private communication.

29. M. K. Liou, R. G. E. Timmermans and B. F. Gibson, Phys. Rev C54 (1996) 1574; Phys. Lett. B345

30. A. Yu. Korchin, O. Scholten and D. Van Neck, Nucl. Phys. A602 (1996) 423.

31. F. de Jong and K. Nakayama, Phys. Lett B385 (1996) 33; Phys. Rev. C52 (1995) 2377.

32. J. A. Eden and M. Gari, Phys. Lett. B347 (1995) 187; Phys. Rev. C53 (1996) 1102.

33. H. O. Meyer et al., Phys. Rev. Lett. 65 (1990) 2846.

34. H. O. Meyer et al., Nucl. Phys. A539 (1992) 633.

35. D. Koltun and A. Reitan, Phys, Rev. 141 (1966) 1413.

36. G. A. Miller and P. U. Sauer, Phys. Rev. C44 (1991) R1775.

37. A. Bondar et al., Phys. Lett. B356 (1995) 8.

38. T. D. Cohen, J. L. Friar, G. A. Miller and U. van Kolck, Phys. Rev. C53 (1996) 2661.

39. B.-Y. Park et al., Phys. Rev. C53 (1996) 1519.

40. T. Sato, T.-S. H. Lee, F. Myrher and K. Kubodera, Phys. Rev. C56 (1997) 1246.

41. U. G. Meissner, Proc. 8th Intl. Conf. Structure of Baryons, nucl-th/98100015.

42. C. Hanhart, J. Haidenbauer, A. Reuber, C. Schütz, and J. Speth, Phys. Lett. B358 
(1995) 21.

43. C. Hanhart, J. Haidenbauer, O. Krehl and J. Speth, Phys. Lett B444 (1-2) (1998) 25.

44. C. Hanhart, J. Haidenbauer, O. Krehl and J. Speth, Phys. Rev. C61 (2000) 064008.

45. V. Dmitrasinovic, K. Kubodera, F. Myhrer and T. Sato, Phys. Lett. B465 (1999) 43.

46. U. van Kolck, G. A. Miller and D. O. Riska, Phys. Lett. B388 (1996) 679.

47. T.-S. H. Lee and D. O. Riska, Phys. Rev. Lett. 70 (1993) 2237.

48. C. J. Horowitz, H. O. Meyer and D. K. Griegel, Phys. Rev. C49 (1994) 1337.

49. J. Adam, A. Stadler, M. T. Peña and F. Gross, Phys. Lett. B407 (1997) 97.

50. E. Hernandéz and E. Oset, Phys. Lett. B350 (1995) 158.

51. H. O. Meyer et al., Phys. Rev. Lett. 81 (1998) 3096.

52. H. O. Meyer et al., Phys. Rev. Lett. 83 (1999) 5939.

53. S. K. Saha et al., Phys. Lett. B461 (1999) 175.

54. J. Zlomańczuk, et al., Phys. Lett. B436 (1998) 251.

55. M. T. Peña, D. O. Riska and A. Stadler, Phys. Rev. C60 (1999) 045201.

56. D. Abbott et al., Phys. Rev. Lett. 84 (2000) 5053.

57. L. C. Alexa et al., Phys. Rev. Lett. 82 (1999) 1374.

58. D. Abbott et al., Phys. Rev. Lett. 82 (1999) 1379.

59. M. van der Schaar et al., Phys. Rev. Lett. 68 (1992) 776.

60. G. van der Steenhoven, Few-Body Syst. 17 (1994) 79.

61. S. J. Wallace, F. Gross, and J. A. Tjon, Phys. Rev. Lett. 74 (1995) 228 ; Phys. Rev. C53 (1996) 860.

62. D. R. Phillips, M. C. Birse and S. J. Wallace, Phys. Rev. C55 (1997) 1937.

63. H. Arenhövel, F. Ritz and T. Wilbois, Phys. Rev. C61 (2000) 034002.

64. F. Ritz, H. Arenhövel and T. Wilbois, Phys. Rev. C55 (1999) 2214; Few Body Systems 24 (1998), 123.

65. E. Hummel and J. A. Tjon, Phys. Rev. Lett. 63 (1989) 1788; Phys. Rev. C42 (1990) 423; Phys. Rev. C49 (1994) 21.

66. J. W. Van Orden, N. Devine and F. Gross, Phys. Rev. Lett. 75 (1995) 4369.

67. D. R. Phillips, S. J. Wallace and N. K. Devine, Phys. Rev. C58 (1998) 2261.

68. D. R. Phillips, S. J. Wallace and N. K. Devine, nucl-th/990686.

69. J. Carbonell and V. A. Karmanov, A663-664 (1-4) (2000) 361.

70. P. L. Chung, F. Coester, B. Keister and W. Polyzou, Phys. Rev. C37 (1988) 2000.

71. F. Gross, J. W. Van Orden and K. Holinde, Phys. Rev. C45 (1992) 2094.

72. M. J. Zuilhof and J. A. Tjon, Phys. Lett. B84 (1979) 31; Phys. Rev. C24 (1981) 736.

73. R. Schiavilla, private communication.

74. N. Isgur and C. H. Llewellyn-Smith, Phys. Rev. Lett. 52 (1984) 1080; Phys. Lett. B217 (1989) 535.

75. A. V. Radyushkin, Acta Phys. Pol. B15 (1984) 403.

76. C. Bochna et al., Phys. Rev. Lett. 81 (1998) 4576.

77. K. Wijesooriya et al. submitted for publication.

78. L. I. Frankfrurt, G. A. Miller, M. M. Sargsian and M. I. Strikman, Phys. Rev. Lett. 84 (2000) 3045. 